\title{
Efficacy and safety of aripiprazole in child and adolescent patients
}

\author{
Eiji Kirino
}

Received: 23 October 2011/Accepted: 14 March 2012/Published online: 25 March 2012

(C) The Author(s) 2012. This article is published with open access at Springerlink.com

\begin{abstract}
Aripiprazole (APZ) has a unique pharmacological profile, as a partial agonist at the dopamine D2 and serotonin 5HT1A receptors and an antagonist at the serotonin 5HT2A receptor; this drug has few side effects (such as extrapyramidal syndrome, hyperprolactinemia, weight gain, metabolic disorders, and sedation) which are typical problems with other antipsychotic drugs. Due to its high tolerability, it is possible to safely administer it to children and adolescents. Efficacy and tolerability of APZ in children and adolescents have been well demonstrated in many clinical studies, which supported approvals granted by the US Food and Drug Administration (FDA) for schizophrenia, bipolar diseases, and irritability associated with autistic disorder in children and adolescents. APZ is expected to exert sedative, anti-depressive, and anti-anxiety effects, and stabilize emotion. APZ is an antipsychotic drug which could be useful for a wider spectrum of psychiatric disorders in children and adolescents. There is little risk of deterioration (such as disinhibition and acting out) and rapid stabilization is easy to achieve in children and adolescents without definitive diagnoses or with a combination of more than one spectrum of disorders. The effectiveness of APZ in children and adolescents is reviewed and discussed, given its pharmacological profile and the outcomes of various
\end{abstract}

\section{E. Kirino}

Department of Psychiatry, School of Medicine,

Juntendo University, Tokyo, Japan

E. Kirino $(\square)$

Department of Psychiatry, Juntendo University Shizuoka Hospital, 1129 Nagaoka, Izunokunishi, Shizuoka 4102211, Japan

e-mail: ekirino@juntendo.ac.jp

E. Kirino

Juntendo Institute of Mental Health, Saitama, Japan clinical studies. However, randomized or blind studies are still limited, and the majority of reports referenced here are open-label studies and case reports. Conclusions drawn from such studies must be evaluated with caution, and a further accumulation of controlled studies is thus needed.

Keywords Aripiprazole · Child · Adolescent · ADHD · Pervasive development disorder · Asperger's syndrome

\section{Introduction}

Aripiprazole (APZ) is an antipsychotic drug approved by the US Food and Drug Administration (FDA) for schizophrenia in 2002. Since then, it has been approved worldwide including Japan in 2006 for treatment of schizophrenia. The efficacy and tolerability of APZ, a dopamine D2 partial agonist [1] distinctive from conventional antipsychotics, have been demonstrated in various psychotic diseases. APZ is indicated in the USA for treatment of schizophrenia and bipolar I disorder, and has been recently approved for the treatment of irritability associated with autistic disorder in children and adolescents. The effectiveness of APZ in children and adolescents is reviewed and discussed herein, given its pharmacological profile and the outcomes of various clinical studies.

\section{Pharmacological profile}

Affinity for dopamine D2 receptors and intrinsic activity

APZ is classified as a third-generation atypical antipsychotic drug because of its unique mechanism of action 
distinctive from that of other atypical antipsychotics [2]. APZ functions as a dopamine D2 partial agonist and appropriates intrinsic activity at dopamine D2 receptors, stabilizing dopamine D2 receptor-mediated neurotransmission without excessive blocking. That is why APZ is often called a dopamine system stabilizer [3]. APZ, which has higher affinity at dopamine D2 receptors than endogenous dopamine, works as a partial agonist. Therefore, APZ is less likely to induce extrapyramidal symptoms (EPS) and elevate serum prolactin levels compared to other antipsychotics. There are a few other partial agonists that have been demonstrated to be effective in reducing negative symptoms and lowering the incidence of EPS, but none of them has exhibited non-inferiority over typical antipsychotics in other psychotic symptoms such as positive symptoms [4-9]. Differences between APZ and other partial agonists can be ascribed to the extent of intrinsic activity at dopamine $\mathrm{D} 2$ receptors. APZ is also a high-affinity partial agonist at dopamine D3 receptors $[4,10]$.

\section{Affinity to serotonin 5HT receptors}

APZ has high affinity to serotonin 5HT1A and 5HT2A receptors. 5HT1A knockout mice exhibit anxiety behavior [11], and 5HT1A receptor agonists appear to have antianxiety and anti-depressive effects and improve cognitive function [12]. APZ is a partial agonist of serotonin 5HT1A, which is expected to improve anxiety and depression and to reduce antipsychotic-derived EPS [13, 14]. de Quervain et al. [15] found that genetic variations of 5HT2A receptors affect human episode memory, which indicates that the 5HT2A receptor is closely related with cognitive function [16]. APZ is a high-affinity antagonist at serotonin 5HT2A receptors, decreasing $5 \mathrm{HT} 2 \mathrm{~A}$ receptor activity, which may improve negative symptoms and reduce antipsychoticsinduced EPS [17].

Wirshing et al. [18] indicated that the second-generation antipsychotics [such as clozapine, risperidone (RIS) and olanzapine (OLZ)] which block serotonin 5HT receptors and histamine receptors are associated with body weight gain. Sargent et al. [19] reported that $m$-chlorophenyl piperazine ( $m$-CPP), a 5HT2C receptor agonist, may continue to activate brain 5-HT2C receptors and this effect is associated with decreases in appetite and body weight. Nasrallah [20] suggested that generic variations of 5HT2C might affect antipsychotics-derived weight gain, because OLZ and RIS significantly increased weight in drug-naïve patients with 5HT2C polymorphism compared with wildtype patients. Therefore, $5 \mathrm{HT} 2 \mathrm{C}$ antagonists are associated with weight gain, while APZ, a 5HT2C receptor partial antagonist, appears to regulate appetite with less effect on weight and an anti-obesity effect.
Affinities for other receptors

Drugs that are high-affinity antagonists at histamine H1 receptors are associated with weight gain or hypersedation [21-23], while high-affinity antagonists at muscaline M1 receptors are related to anticholinergic side effects such as dry mouth, constipation, and blurred vision [23-25]. APZ is an antagonist, but has a low affinity at histamine H1, adrenaline $\alpha 1$ [21], and muscaline M1 receptors [22] and few side effects [26], as described in the guidelines of American Psychiatric Association (APA) [27].

\section{Efficacy in children and adolescents}

APZ is indicated in the USA for acute and maintenance treatment of schizophrenia in adolescents (13-17 years of age) and acute treatment of bipolar I disorder in children (10-17 years of age) as monotherapy and as an adjunctive to lithium or valproate. It is also indicated for use in treating irritability associated with autistic disorder in pediatric patients (6-17 years of age) [28-32]. Aparasu et al. [33] examined patterns and determinants of antipsychotic prescribing in children and adolescents receiving outpatient care in the USA. They analyzed outpatient visits involving 11 typical and 6 atypical antipsychotic agents prescribed for children and adolescents. Most (99\%) of these visits involved prescribing of atypical agents, and the most frequently prescribed atypical agents were RIS, QTP, and APZ.

\section{Schizophrenia}

Findling et al. [34] reported the results of a 6-week, multicenter, double-blind, randomized, placebo-controlled trial in which children and adolescents (13-17 years of age, $n=302$ ) with schizophrenia and a PANSS total score of 70 or more were randomly assigned (1:1:1 ratio) to placebo, $10 \mathrm{mg} /$ day of APZ, or $30 \mathrm{mg} /$ day of APZ. At the end of the study, both APZ doses showed statistically significant differences from placebo in reduction in PANSS total score and were well tolerated in all patients.

Kirino [35] reported that APZ was effective in a 15-year-old schizophrenic patient with long-term catatonic stupor. This patient was first given RIS $0.5-2 \mathrm{mg} / \mathrm{day}$ and switched to APZ $6 \mathrm{mg} /$ day due to hypersedation. After the APZ dose was increased to $12 \mathrm{mg} /$ day, the patient started to speak more; he had more active communications after the dose was increased to $18 \mathrm{mg} /$ day without any adverse events. This case suggests a unique effect of APZ as a dopamine D2 receptor partial agonist distinctive from other antipsychotics, with reduced risk of hypersedation, and as a serotonin 5HT1A receptor partial agonist that is useful in 
treating catatonic anxiety. APZ may be effective in the treatment of some schizophrenic patients with stupor, which sometimes carries a risk of physical debilitation and requires special attention due to the risk of adverse drug reactions.

\section{Bipolar I disorder}

Tramontina et al. [36] assessed the response to treatment with APZ in children and adolescents with bipolar disorder comorbid with attention-deficit/hyperactivity disorder (ADHD) in a 6-week double-blind, placebo-controlled trial. The group receiving APZ (8-17 years of age, $n=18$ ) showed a significantly greater reduction in the Young Mania Rating Scale (YMRS) scores, the Child Mania Rating Scale-Parental Version (CMRS-P) scores, and the Clinical Global Impressions-Severity (CGI-S) scores from baseline to end point when compared with the placebo group $(n=25)$. In addition, higher rates of response and remission were found for the APZ group. No significant between-group differences were found in weight, ADHD symptoms, or depressive symptoms. Adverse events significantly more frequent in the APZ group were somnolence and sialorrhea. APZ was effective in reducing manic symptoms and improving global functioning without promoting severe adverse events or weight gain. However, conclusions drawn from such a study including subjects with comorbid condition must be evaluated with caution.

Findling et al. [30] carried out a randomized, doubleblind, placebo-controlled trial with a follow-up period of 4 weeks in 296 cases aged 10-17 years with bipolar disorder type I (mania) and with a mixed episode. They reported that APZ $10 \mathrm{mg} /$ day or $30 \mathrm{mg} /$ day significantly improved the YMRS total score by 1 week and the response rate (a reduction in the YMRS total score by $50 \%$ or more) 4 weeks later was significantly higher than in the placebo cases; this suggested that APZ was effective for bipolar disorder at the acute stage in childhood and adolescence.

\section{Pervasive development disorder (PDD)}

In an 8-week double-blind, randomized, placebo-controlled, parallel-group study reported by Marcus et al., 218 children and adolescents (6-17 years of age) with autistic disorder and behaviors such as tantrums, aggression, selfinjurious behavior, or a combination of these symptoms, were randomized 1:1:1:1 to $\mathrm{APZ}(5,10$, or $15 \mathrm{mg} /$ day $)$ or placebo. All APZ doses demonstrated significantly greater improvements than placebo in mean Aberrant Behavior Checklist Irritability (ABC-I) subscale scores and mean CGI-I score than placebo at week 8 . The most common adverse event leading to discontinuation was sedation. There were two serious adverse events: presyncope ( $5 \mathrm{mg} / \mathrm{day})$ and aggression (10 mg/day). At week 8 , mean weight change was as follows: placebo $+0.3 \mathrm{~kg}$, APZ $5 \mathrm{mg} /$ day $+1.3 \mathrm{~kg}$, APZ $10 \mathrm{mg} /$ day $+1.3 \mathrm{~kg}$, and APZ $15 \mathrm{mg} /$ day $+1.5 \mathrm{~kg}$; all $p<0.05$ versus placebo. APZ was efficacious and generally safe and well tolerated in the treatment of children and adolescents with irritability associated with autistic disorder [37].

In a 14-week, prospective, open-label study [38], APZ was administered to 25 children and adolescents (5-17 years of age) diagnosed with pervasive developmental disorder not otherwise specified (PDD-NOS) or Asperger's syndrome (range: $2.5-15.0 \mathrm{mg} / \mathrm{day}$, mean dose: $7.8 \mathrm{mg} /$ day). Full-scale intelligence quotient (IQ) scores ranged from 48 to 122 (mean 84). Twenty-two of 25 subjects (88\%) were responders with regard to interfering symptoms of irritability, including aggression, self-injury, and tantrums, with a final CGI-I of 1 or 2 (very much or much improved) and a $25 \%$ or greater improvement on the Aberrant Behavior Checklist (ABC-I). The final mean CGI-I was 1.6. ABC-I scores ranged from 18 to 43 (mean 29) at baseline, whereas scores at week 14 ranged from 0 to 27 (mean 8.1). APZ was well tolerated. Mild EPSs were reported in nine subjects (36\%). Body mass index (BMI) increased from 20.3 at baseline to 21.1 at end point. Prolactin significantly decreased from 9.3 to $2.9 \mathrm{ng} / \mathrm{mL}$. These data suggest that APZ may be effective and well tolerated for severe irritability in pediatric patients with PDD-NOS or Asperger's syndrome. In a retrospective naturalistic study in 34 patients (4.5-15 years of age) with severely impaired PDD [39], the mean baseline CGI-S was 5.7 (markedly ill/severely ill). The mean final dosage of APZ was $8.1 \mathrm{mg} /$ day. At the end point, 11 patients $(32.4 \%)$ were 'much improved' or 'very much improved' (CGI-I score of 1 or 2$), 12$ patients $(35.3 \%)$ were 'minimally improved' (CGI-I score of 3), and 10 (29.4\%) were 'unchanged' or 'worsened' (CGI-I score of 4 or 5). The Children's Global Assessment Scale (C-GAS) and the Childhood Autism Rating Scale (CARS) scores significantly improved. Nine patients $(26.5 \%)$ experienced moderate to severe agitation, which was associated with self-injurious behaviors in five of these patients, and five patients $(14.7 \%)$ presented with sleep disorders. Twelve patients $(35.3 \%)$ discontinued medication during the follow-up because of lack of efficacy or adverse effects. In these severely impaired children with PDDs, APZ monotherapy was associated with a significant improvement in maladaptive behaviors in one-third of patients. Agitation and insomnia were the most frequent adverse effects.

Attention-deficit hyperactivity disorder (ADHD)

In a 6-week open-label study in ADHD patients (combined type and predominately inattentive type) (8-12 years of 
age, $N=23$ ) [40], end of study results showed overall significant improvement from baseline on ADHD and functional outcome measures at a mean dose of $6.7 \mathrm{mg} /$ day. No significant differences from baseline performance were found on the cognitive measures at the end of the study. The most frequently reported adverse events were sedation $(78.3 \%)$ and headache $(47.8 \%)$. In this cohort, APZ led to clinical benefit in reducing ADHD symptoms and improving overall functioning. Of note, cognitive functioning did not appear to be negatively impacted by APZ treatment.

Tic disorders

Yoo et al. [41] investigated the efficacy and tolerability of APZ for treating children and adolescents with tic disorders in an 8-week open-label study using a flexible dosing schedule (range: $2.5-20 \mathrm{mg} /$ day, mean dose: $9.8 \mathrm{mg} /$ day) in 24 outpatients (7-18 years of age). There was a $52.5 \%$ reduction in the mean score of the Korean versions of the Yale Global Tic Severity Scale (YGTSS) Total Tic scores. Nineteen patients $(79.2 \%)$ showed either much improved or very much improved status according to the CGI-I. The CGI-S score was also reduced (from 5.5 to 3.0). The initial dose of $5 \mathrm{mg} /$ day APZ for 2 weeks was also found to reduce tic symptoms significantly. This study suggests that APZ is an efficacious and safe treatment for children and adolescents with tic disorders. Seo et al. [42] conducted a study to evaluate the effectiveness of APZ to reduce the severity and frequency of tic symptoms and to evaluate the additional effects of APZ on weight changes in children and adolescents with Tourette's syndrome or chronic tic disorders. A 12-week, open-label trial with flexible dosing strategy of APZ was performed with 15 participants (7-19 years of age). Significant decreases in the scores of motor and phonic tics, global impairment, and global severity were demonstrated between baseline and week 3 , and the scores continued to improve thereafter. No difference was observed between the baseline and end point body mass index (BMI) values. This study demonstrates that a relatively low dose of APZ can be used to control tic symptoms effectively in children and adolescents with Tourette's syndrome and chronic tic disorders without causing significant weight gain.

\section{Delusional disorders}

Myers et al. [43] treated a 17-year-old female diagnosed with delusional disorder (erotomanic type with a history of seven episodes of stalking) with APZ $10 \mathrm{mg} /$ day combined with cognitive behavior therapy and family therapy. As a result of treatment, she became better able to communicate with others, with continued improvement at month 5 , and was able to avoid a contact with the stalking victim. She is still being treated with APZ $10 \mathrm{mg} /$ day; the drug has been well tolerated. They concluded that combination treatment with APZ and psychological therapy are effective in addressing delusional disorders.

\section{Obsessive-compulsive disorder (OCD)}

Storch et al. [44] reported a 13-year-old male with OCD who had an incomplete response to combined cognitivebehavioral therapy (CBT) and sertraline before successful augmentation of CBT with APZ 2.5-5 mg/day. Standardized assessments indicated significant reductions in OCD symptomatology associated with both initial treatment and APZ augmentation. This case suggests that APZ may have utility as an augmenting agent of CBT in adolescents with OCD by virtue of its dual impact on serotonergic and dopaminergic mechanisms.

Reported clinical and safety outcomes of APZ in children and adolescents referenced above are summarized in Table 1.

\section{Safety data in children and adolescents}

Body weight and metabolic parameters

Correll et al. [45] carried out a cohort study to evaluate cardiometabolic effects of the second-generation antipsychotics in children and adolescents (4-19 years of age) naive to antipsychotic medication. After a median of 10.8 weeks of treatment, mean body weight increased by $8.5 \mathrm{~kg}$ with OLZ $(n=45), 6.1 \mathrm{~kg}$ with QTP $(n=36)$, $5.3 \mathrm{~kg}$ with RIS $(n=135)$, and $4.4 \mathrm{~kg}$ with APZ $(n=41)$ compared with the minimal weight change of $0.2 \mathrm{~kg}$ in the untreated comparison group $(n=15)$. With OLZ and QTP, respectively, the mean levels increased significantly for total cholesterol (15.6-24.3 and 9.1-17.7 mg/dL), triglycerides $(24.3-38.9$ and $37.0-63.8 \mathrm{mg} / \mathrm{dL})$, non-HDL cholesterol [total cholesterol minus HDL cholesterol] (16.8-24.3 and 9.9-18.4 mg/dL), and the ratio of triglycerides to HDL cholesterol (0.6-0.9 and 1.2-2.0). With RIS, triglycerides increased significantly $(9.7-19.0 \mathrm{mg} / \mathrm{dL})$. Metabolic baseline-to-endpoint changes were not significant with APZ or in the untreated comparison group.

EPS and akathisia

In randomized, double-blinded, placebo-controlled trials of schizophrenia [34], bipolar type I disorder [30], and autism $[31,37]$ in childhood and adolescence, it was reported that the occurrence of EPS was more frequent in the APZ group than in the placebo group and salivation, tremor, and 


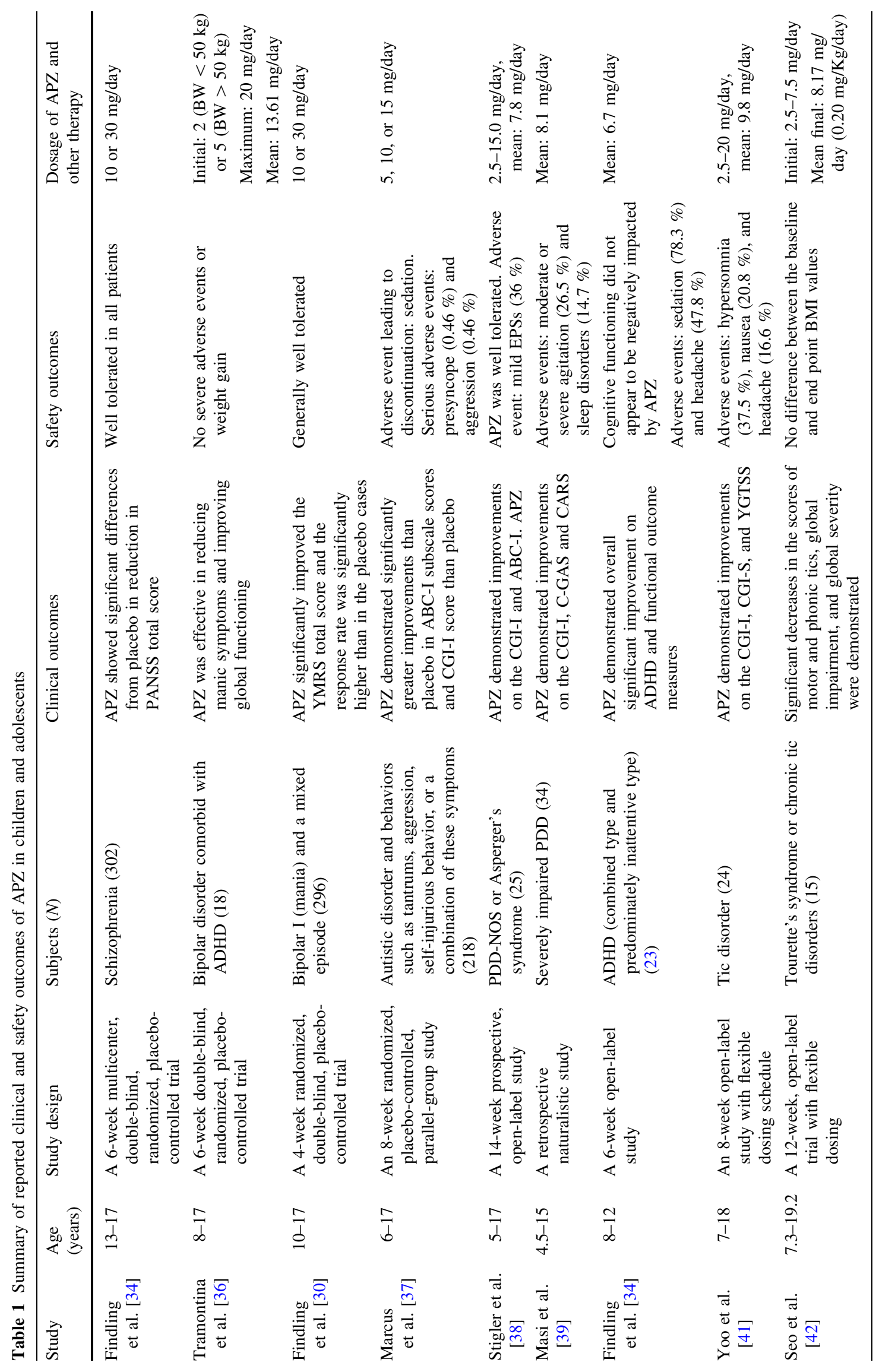


dystonia were observed. However, the degree of EPS was considered mild to moderate.

Akathisia was more frequently observed in the highdose APZ group ( $30 \mathrm{mg} /$ day), but it was comparably observed between the group receiving APZ at $10 \mathrm{mg} /$ day and the placebo group [34]. Findling et al. [30] reported that the occurrence rate of akathisia was higher in the group of patients receiving APZ $10 \mathrm{mg} /$ day or greater when compared with the control group. Moreover, there was another report that the occurrence rate was comparable between the APZ and placebo groups [31, 37]. Therefore, no consensus has been reached as to the occurrence rate of akathisia in patients taking APZ.

However, since the approved dose of APZ is 10 or $30 \mathrm{mg} /$ day for schizophrenia and bipolar disorder cases and 5,10 , or $15 \mathrm{mg} /$ day for autism cases, there is a possibility that the occurrence rate of akathisia is higher in cases receiving a higher dose of APZ.

\section{Sedation}

Among the randomized, double-blinded, placebo-controlled trials, sedation was more frequently observed in the APZ group than in the placebo group in trials on autism $[31,37]$. No adverse event of sedation was reported in cases with schizophrenia [34] and bipolar disorder [30], but the sleepiness appeared more frequently in the APZ group than in the placebo group. It was considered that a higher dose of APZ induced a higher rate of sedation and sleepiness in cases with these disorders.

\section{Serum prolactin}

The serum prolactin level was significantly lower after APZ administration than at baseline, and it was also significantly lower in the APZ group than in the placebo group in the randomized, double-blinded, placebo-controlled trials for schizophrenia [34], bipolar type I disorder [30], and autism [31, 37].

\section{Other parameters}

There were no changes evident in blood pressure, heart rates, ECG, or the QTc interval in any of the doubleblinded, placebo-controlled trials [31, 34, 37].

\section{Conclusion}

APZ is expected to exert sedative, anti-depressive, and anti-anxiety effects, and to stabilize emotion. Due to its high tolerability, it is possible to safely administer it to children and adolescents. There is little risk of deterioration (such as disinhibition and acting out) and rapid stabilization is easy to achieve in children and adolescents without definitive diagnoses or with a combination of more than one spectrum of disorders. In particular, since the safety of antidepressants is not established in child and adolescents, the utility of the antidepressant effect of APZ is high. It is possible to make quality of life (QOL) improvement treatment targets with APZ administration, as well as positive symptoms in cases of irritability, aggression, anxiety, or depression.

The expert consensus guidelines on adherence problems in patients with mental illness published in 2009 discussed the side effects of medication as a factor in poor adherence to medication treatment; in particular, weight gain, sedation, akathisia, and sexual dysfunction were considered important factors leading to adherence problems [46]. Therefore, medication with a lower risk of these side effects appears to have a great impact on patient outcomes. The use of an antipsychotic agent with fewer side effects might be more critical in children and adolescents than adults to allow for continued treatment. APZ has few side effects (such as EPS, hyperprolactinemia, weight gain, metabolic disorders, and sedation) which are typical problems with other antipsychotic drugs. Furthermore, the ameliorative effects of APZ on cognitive function and communication ability consequently led to improvement of QOL. The efficacy and tolerability of APZ in children and adolescents have been well demonstrated in many clinical studies. APZ is an antipsychotic drug which could be useful for a wider spectrum of psychiatric disorders in children and adolescents. However, randomized or blind studies are still limited, and the majority of reports referenced here are open-label studies and case reports. Conclusions drawn from such studies must be evaluated with caution, and further accumulation of controlled studies is thus needed.

\section{Conflict of interest None.}

Open Access This article is distributed under the terms of the Creative Commons Attribution License which permits any use, distribution, and reproduction in any medium, provided the original author(s) and the source are credited.

\section{References}

1. Kikuchi $\mathrm{T}$ (2007) The research and development of aripiprazole and its mechanism of action. Jpn J Clin Psychopharmacol 10(3):464-468

2. Stahl SM (2001) Dopamine system stabilizers, aripiprazole, and the next generation of antipsychotics, part 2: illustrating their mechanism of action. J Clin Psychiatry 62(12):923-924

3. Tadori Y, Miwa T, Tottori K, Burris KD, Stark A, Mori T et al (2005) Aripiprazole's low intrinsic activities at human dopamine 
D2L and D2S receptors render it a unique antipsychotic. Eur $\mathbf{J}$ Pharmacol 515(1-3):10-19

4. Potkin SG, Saha AR, Kujawa MJ, Carson WH, Ali M, Stock E et al (2003) Aripiprazole, an antipsychotic with a novel mechanism of action, and risperidone vs placebo in patients with schizophrenia and schizoaffective disorder. Arch Gen Psychiatry 60(7):681-690

5. Lahti AC, Weiler MA, Corey PK, Lahti RA, Carlsson A, Tamminga CA (1998) Antipsychotic properties of the partial dopamine agonist (-)-3-(3-hydroxyphenyl)- $N$ - $n$-propylpiperidine (preclamol) in schizophrenia. Biol Psychiatry (Clinical Trial Controlled Clinical Trial Research Support, U.S. Gov't, P.H.S.) 43(1):2-11

6. Tamminga CA, Carlsson A (2002) Partial dopamine agonists and dopaminergic stabilizers, in the treatment of psychosis. Curr Drug Targets CNS Neurol Disord 1(2):141-147 (Review)

7. Tamminga CA (2002) Partial dopamine agonists in the treatment of psychosis. J Neural Transm 109(3):411-420 (Review)

8. Olbrich R, Schanz H (1991) An evaluation of the partial dopamine agonist terguride regarding positive symptoms reduction in schizophrenics. J Neural Transm Gen Sect. (Clinical Trial) 84(3): 233-236

9. Olbrich R, Schanz H (1988) The effect of the partial dopamine agonist terguride on negative symptoms in schizophrenics. Pharmacopsychiatry 21(6):389-390

10. Shapiro DA, Renock S, Arrington E, Chiodo LA, Liu LX, Sibley DR et al (2003) Aripiprazole, a novel atypical antipsychotic drug with a unique and robust pharmacology. Neuropsychopharmacology 28(8):1400-1411

11. Heisler LK, Chu HM, Brennan TJ, Danao JA, Bajwa P, Parsons LH et al (1998) Elevated anxiety and antidepressant-like responses in serotonin 5-HT1A receptor mutant mice. Proc Natl Acad Sci USA 95(25):15049-15054

12. Meltzer HY, Li Z, Kaneda Y, Ichikawa J (2003) Serotonin receptors: their key role in drugs to treat schizophrenia. Prog Neuropsychopharmacol Biol Psychiatry 27(7):1159-1172

13. DeBattista C, Hawkins J (2009) Utility of atypical antipsychotics in the treatment of resistant unipolar depression. CNS Drugs 23(5):369-377

14. Fernandez HH, Trieschmann ME, Friedman JH (2004) Aripiprazole for drug-induced psychosis in Parkinson disease: preliminary experience. Clin Neuropharmacol 27(1):4-5

15. de Quervain DJ, Henke K, Aerni A, Coluccia D, Wollmer MA, Hock C et al (2003) A functional genetic variation of the 5-HT2a receptor affects human memory. Nat Neurosci 6(11):1141-1142

16. Grunder G, Kungel M, Ebrecht M, Gorocs T, Modell S (2006) Aripiprazole: pharmacodynamics of a dopamine partial agonist for the treatment of schizophrenia. Pharmacopsychiatry 39(Suppl 1):S21-S25

17. Hirose T, Uwahodo Y, Yamada S, Miwa T, Kikuchi T, Kitagawa $\mathrm{H}$ et al (2004) Mechanism of action of aripiprazole predicts clinical efficacy and a favourable side-effect profile. J Psychopharmacol 18(3):375-383

18. Wirshing DA, Boyd JA, Meng LR, Ballon JS, Marder SR, Wirshing WC (2002) The effects of novel antipsychotics on glucose and lipid levels. J Clin Psychiatry 63(10):856-865

19. Sargent PA, Sharpley AL, Williams C, Goodall EM, Cowen PJ (1997) 5-HT2C receptor activation decreases appetite and body weight in obese subjects. Psychopharmacology 133(3):309-312

20. Nasrallah HA (2008) Atypical antipsychotic-induced metabolic side effects: insights from receptor-binding profiles. Mol Psychiatry 13(1):27-35

21. Kroeze WK, Hufeisen SJ, Popadak BA, Renock SM, Steinberg S, Ernsberger P, et al (2003) H1-histamine receptor affinity predicts short-term weight gain for typical and atypical antipsychotic drugs. Neuropsychopharmacology (Comparative Study Research
Support, Non-U.S. Gov't Research Support, U.S. Gov't, P.H.S.) 28(3):519-526

22. Goodnick PJ, Jerry JM (2002) Aripiprazole: profile on efficacy and safety. Expert Opin Pharmacother 3(12):1773-1781

23. Stahl SM, Shayegan DK (2003) The psychopharmacology of ziprasidone: receptor-binding properties and real-world psychiatric practice. J Clin Psychiatry 64(Suppl 19):6-12

24. Bolden C, Cusack B, Richelson E (1992) Antagonism by antimuscarinic and neuroleptic compounds at the five cloned human muscarinic cholinergic receptors expressed in Chinese hamster ovary cells. J Pharmacol Exp Ther (Research Support, Non-U.S. Gov't Research Support, U.S. Gov't, P.H.S.) 260(2):576-580

25. Shayegan DK, Stahl SM (2004) Atypical antipsychotics: matching receptor profile to individual patient's clinical profile. CNS Spectr 9(10 Suppl 11):6-14

26. Davies MA, Sheffler DJ, Roth BL (2004) Aripiprazole: a novel atypical antipsychotic drug with a uniquely robust pharmacology. CNS Drug Rev 10(4):317-336

27. American Psychiatric Association (2004) Practice guideline for the treatment of patients with schizophrenia, 2nd edn. American Psychiatric Association, Arlington (VA)

28. Sanford M, Keating GM (2007) Aripiprazole: in adolescents with schizophrenia. Paediatr Drugs 9(6):419-423

29. Biederman J, Mick E, Spencer T, Doyle R, Joshi G, Hammerness $P$ et al (2007) An open-label trial of aripiprazole monotherapy in children and adolescents with bipolar disorder. CNS Spectr 12(9):683-689

30. Findling RL, Nyilas M, Forbes RA, McQuade RD, Jin N, Iwamoto $\mathrm{T}$ et al (2009) Acute treatment of pediatric bipolar I disorder, manic or mixed episode, with aripiprazole: a randomized, double-blind, placebo-controlled study. J Clin Psychiatry 70(10):1441-1451

31. Owen R, Sikich L, Marcus RN, Corey-Lisle P, Manos G, McQuade RD et al (2009) Aripiprazole in the treatment of irritability in children and adolescents with autistic disorder. Pediatrics 124(6): 1533-1540

32. Robb AS, Carson WH, Nyilas M, Ali M, Forbes RA, Iwamoto T et al (2010) Changes in positive and negative syndrome scalederived hostility factor in adolescents with schizophrenia treated with aripiprazole: post hoc analysis of randomized clinical trial data. J Child Adolesc Psychopharmacol 20(1):33-38

33. Aparasu RR, Bhatara V (2007) Patterns and determinants of antipsychotic prescribing in children and adolescents, 2003-2004. Curr Med Res Opin 23(1):49-56

34. Findling RL, Robb A, Nyilas M, Forbes RA, Jin N, Ivanova S et al (2008) A multiple-center, randomized, double-blind, placebo-controlled study of oral aripiprazole for treatment of adolescents with schizophrenia. Am J Psychiatry 165(11):14321441

35. Kirino E (2010) Prolonged catatonic stupor successfully treated with aripiprazole in an adolescent male with schizophrenia: a case report. Clin Schizophr Relat Psychoses 4(3):185-188

36. Tramontina S, Zeni CP, Ketzer CR, Pheula GF, Narvaez J, Rohde LA (2009) Aripiprazole in children and adolescents with bipolar disorder comorbid with attention-deficit/hyperactivity disorder: a pilot randomized clinical trial. J Clin Psychiatry 70(5):756-764

37. Marcus RN, Owen R, Kamen L, Manos G, McQuade RD, Carson WH et al (2009) A placebo-controlled, fixed-dose study of aripiprazole in children and adolescents with irritability associated with autistic disorder. J Am Acad Child Adolesc Psychiatry 48(11):1110-1119

38. Stigler KA, Diener JT, Kohn AE, Li L, Erickson CA, Posey DJ et al (2009) Aripiprazole in pervasive developmental disorder not otherwise specified and Asperger's disorder: a 14-week, prospective, open-label study. J Child Adolesc Psychopharmacol 19(3):265-274 
39. Masi G, Cosenza A, Millepiedi S, Muratori F, Pari C, Salvadori F (2009) Aripiprazole monotherapy in children and young adolescents with pervasive developmental disorders: a retrospective study. CNS Drugs 23(6):511-521

40. Findling RL, Short EJ, Leskovec T, Townsend LD, Demeter CA, McNamara NK et al (2008) Aripiprazole in children with attention-deficit/hyperactivity disorder. J Child Adolesc Psychopharmacol 18(4):347-354

41. Yoo HK, Choi SH, Park S, Wang HR, Hong JP, Kim CY (2007) An open-label study of the efficacy and tolerability of aripiprazole for children and adolescents with tic disorders. J Clin Psychiatry 68(7):1088-1093

42. Seo WS, Sung HM, Sea HS, Bai DS (2008) Aripiprazole treatment of children and adolescents with Tourette disorder or chronic tic disorder. J Child Adolesc Psychopharmacol 18(2):197-205
43. Myers WC, Ruiz R (2004) Aripiprazole and psychotherapy for delusional disorder, erotomanic type. J Am Acad Child Adolesc Psychiatry 43(9):1069-1070

44. Storch EA, Lehmkuhl H, Geffken GR, Touchton A, Murphy TK (2008) Aripiprazole augmentation of incomplete treatment response in an adolescent male with obsessive-compulsive disorder. Depress Anxiety 25(2):172-174

45. Correll CU, Manu P, Olshanskiy V, Napolitano B, Kane JM, Malhotra AK (2009) Cardiometabolic risk of second-generation antipsychotic medications during first-time use in children and adolescents. JAMA 302(16):1765-1773

46. Velligan DI, Weiden PJ, Sajatovic M, Scott J, Carpenter D, Ross $\mathrm{R}$, et al (2009) The expert consensus guideline series: adherence problems in patients with serious and persistent mental illness. J Clin Psychiatry 70(Suppl 4):1-46 (quiz 7-8) 